\title{
1
}

\section{ATOMIC MASS MEASUREMENTS WITH RADIOACTIVE BEAMS AND/OR TARGETS: WHERE TO START}

\author{
Peter E. Haustein \\ Department of Chemistry \\ Brookhaven National Laboratory \\ Upton, New York 11973 USA
}

BNL --42573

DE90 001532

\subsection{ABSTRACT}

Radioactive beams or radioactive targets (or both) can significantly increase the yields of exotic isotopes, allowing studies to be performed in regions which are currently inaccessible. An important goal to pursue with these exotic species is a broad program of nuclidic mass measurements. This is motivated by the observation that mass model predictions generally diverge from one another in regions far from beta-decay stability where well measured masses are sparse or nonexistant. Stringent tests of mass models are therefore possible and these can highlight important features in the mass models that affect the quality of their short-range and long-range extrapolation properties. Selection of systems to study can be guided, in part, by a desire to probe those regions where distinctions among mass models are most apparent and where exotic isotope yields will be optimal. Several examples will be presented to highlight future opportunities in this area.

\section{I.2 INTRODUCTION}

Nuclei far from stability have traditionally been a fertile ground for tests of models of nuclear structure, nuclear masses, and for the discovery of new nuclear decay modes and structural dynamics. Several illustrative examples are: (super)deformation regions and shape evolution; fissility variation and fission modes; beta-delayed particle emission; direct proton radioactivity; synthesis of the heaviest elements; and the interplay between nuclear decay properties and r-and $r$-process nucleosynthesis.

One can confidently expect this to continue as new methods that employ radioactive beams and/or radioactive targets allow even greater excursions to be made from the valley of beta stability. Indeed, the increase of available decay energy alone opens more exotic decay channnels, many of which may prove to be the doorways to the study of new features of nuclear structure.

It is instructive to look in detail at one selected area -- masses of nuclei far from stability. This is motivated by the fact that mass measurements of new nuclei provide stringent tests of mass models. These new nuclei were not part of the database which had been used to refine the parameters used in the construction of the mass model. Therefore well measured masses of these new nuclides serve as inpartial tests of the predictive quality among diverse sets of mass models. Such tests frequently serve to identify "good" mass models, i.e. those models whose predictions on either a global basis or in restricted mass regions prove to be of highest quality. Having identified a model (or models) in this way, the selection of additional experiments can be guided with greater assurance.

\subsection{SHORT REVIEW OF MASS MODELS}

A short review of the methods used in the construction of mass models is useful in the context of the analysis which will follow. A more complete description of methods can be found in the most recent 


\section{DISCLAIMER}

This report was prepared as an account of work sponsored by an agency of the United States Government. Neither the United States Government nor any agency thereof, nor any of their employees, makes any warranty, express or implied, or assumes any legal liability or responsibility for the accuracy, completeness, or usefulness of any information, apparatus, product, or process discloced, or represents that its use would not infringe privately owned rights. Referenci herein to any specific commercial product, process, or service by trade name, trademart, manufacturer, or otherwise does not necessarily constitute or imply its endorsement, recommendation, or favoring by the United States Government or any atency thereof. The views and opinions of authors expressed herein do not necessarily state or reflect those of the United States Government or any agency thereof. 
compilation of mass model predictions and related material 1 . Construction of a mass model generally involves several steps. The model's basic content is first selected, i.e. ab-initio, phenomenological, semi-empirical, liquid drop(let), shell model, or mass-relation based. The next step is the selection and evaluation of each mathematical component of the model that is thought to characterize the relevant physical features of the mass surface. From this step a prototype mass equation is generated. The third step involves attempts to fit the prototype mass equations to the known mass surface. This generally involves the use of iterative least-squares methods wherein mass equation terms and their coefficients are adjusted to minimize residuals with respect to the body of known masses. Once "best fit" parameters are obtained the final step consists of the calculation of predicted masses for the measured (input) masses and the calculation of predicted (output) masses for isotopes beyond the known mass surface. This is done generally out to the neutron and proton drip-lines and into the region of superheavy elements, unless features of the model preclude such calculations or there are reasons to believe that the predictions become intrinsically unreliable in these regions.

\subsection{CHARACTERISTICS OF MASS MODELS IN REGIONS WHERE EXPERIMENTAL DATA EXIST}

The "goodness of fit" of a particular model can be quantified by the average and root-mean-square (rms) deviations of the predicted masses relative to the known masses. Average deviation indicates whether, on a global basis, the calculation has predicted slight over-binding or slight under-binding relative to the isotopic database. RMS deviation signals the degree of conformation of the predicted mass surface to the measured one. In general smaller rms deviations are achieved through the use of larger numbers of adjustable parameters in the models. An interesting correlation between the numbers of adjustable parameters in mass models and the rms deviations of these models to the known mass surface was noted by Tondeur 2 . He observed that models which have most successfully and proficientiy incorporated the relevant physical features of mass surface achieve the smallest rms deviations with a minimum number of adjustable parameters.

In the 1986 - 1987 Atomic Mass Predictions ${ }^{1}$ residuals for each mass model were' plotted in the format of the Chart of the Nuclides. This allows one to gauge the reliability of the predictions in regions that border the experimentally known surface. The smallest collections of residuals are generally associated with those models employing the largest number of adjustable parameters. This does not necessarily mean that such models will have the best extrapolation properties. Analyses 3-5 of earlier (1975 - 81) sets of predictions 6-9 showed that for those models there is only a rough correlation between the quality of fits to the known mass surface and the quality of mass predictions for new isotopes that were subsequently measured.

\subsection{RADIOACTIVE BEAMS AND TARGETS IN THE PRODUCTION OF NEW EXOTIC ISOTOPES}

It is anticipated that one or more large national research centers will be established to provide users with access to radioactive beam facilities. The use of these radioactive beams (possibly in combination with radioactive targets) will permit the production of many new exotic nuclear species with yields that will be substantially higher than are obtainable with more conventional production methods. For example, fusion-evaporation or deep inelastic scattering reactions could start from projectile-target combinations where the reaction partners possess $N / Z$ ratios that are significantly different from those of stable nuclides. This feature and careful control of reaction excitation energies can dramatically increase the yields of exotic isotopes far from stability. Projectile fragmentation of medium-energy to high-energy heavy-ion beams is known to yield neutron-rich species. Capture, deceleration, and cooling of such fragments will yield neutron-rich secondary beams. Nuclear structure information can be obtained directly from these beams or reactions of such beams with stable 
relatively neutron-rich targets will provide large improvements in the yields of new neutron-rich species. Even larger yields will be possible when radioactive targets that are even more neutron rich are used. Eventual synthesis of the postulated superheavy elements may be achieved this way.

\subsection{CHARACTERISTICS OF MASS MODELS IN REGIONS WHERE DATA DO NOT PRESENTLY EXIST}

It is instructive to examine the predictions of atomic mass models in regions beyond the body of measured masses, since these regions will be the ones where exploratory studies with radioactive beams will begin. Previous experience has shown that mass model predictions (as well as predictions of other nuclear properties) diverge from one another in regions far from stability and that this divergence frequently becomes large and occurs rapidly on excursions from the valley of beta stability. Where these divergencies occur and how fast they occur are useful pieces of information. They can point to specific mass regions where selected projectile/target combinations can be employed to produce optimal yields of new isotopes for broad ranging studies of nuclear structure and masses at those places where distinctions among models are most apparent.

For the specific case of the divergencies in mass model predictions in regions far from stability, a detailed analysis of the 1986 - 1987 Atomic Mass Predictions has been made. Two sets of predictions (Pape \& Antony and Dussel, Caurier, and Zuker) do not contain predictions that cover large regions far from stability. Of the remaining eight sets of predictions (Möller-Nix, Möller et al, Comay et al., Satpathy-Nayak, Tachibana et al., Spanier-Johannson, Jänecke-Masson, and MassonJänecke) each includes predictions on both the proton-rich and neutron-rich sides of stability from the lightest elements up to the heaviest actinides (Spanier-Johannson predict only for Z > 51).

Root-mean-square divergencies in the mass predictions were computed using these eight sets of calculations in the following way: 1) predictions for any isotope (measured or predicted by systematics) in the 1986 Wapstra-Audi-Hoekstra mass table were excluded; 2) any isotope which had been predicted by any model to be on or beyond the proton or neutron drip lines was excluded; and 3) at least six predictions had to exist for the remaining cases (approximately 1,100 nuclides beyond the known mass surface) -- in most cases seven or all eight predictions were used. Once the rms deviations of the predictions were calculated they were binned according to size and plotted (Figures $1-5$ ) in the format of the chart of the nuclides.

Two additional levels of analyses were then employed. In the first the plots were inspected to identify trends, i.e. the size and rate of the spread of the mass predictions. The trends were divided intc iwo classes. The first was where the divergencies in the mass model predictions tended to be small and to grow slowly. The second consisted of mass regions with large and/or rapidly growing divergencies in the mass predictions. Results of these analyses were then used in a final analysis step to identify appropriate (radioactive) beam/(radioactive) target combinations to reach these mass regions.

\subsubsection{Regions where divergencies in mass model predictions are small}

Several regions where the divergencies in mass model predictions are small have been identified. While it may be comforting that a consensus exists among different sets of predictions in these regions, it is important nevertheless to perform experimental tests of theory in each of these regions. These tests can generally be made with modest extensions of existing methodologies; they will not require the more "heroic" efforts outline below in section 1.6.2. Also, in some cases the origins of the small divergencies among the mass models can be rationalized. Each case will be examined in order of increasing mass. 
$\underline{Z}=27-29$, neutron rich; The first case is show in figure 1 with the cluster of squares, signaling smallest rms deviations between zero and $0.5 \mathrm{MeV}$, located for neutron-rich nuclei with $\mathrm{Z}=27-29$, just beyond the known mass surface. This case seems to be clearly associated with the proton shell closure at $Z=28$ and implies that the mass models which contain quite diverse "physics" nonetheless yield nearly identical predictions. It is also quite probable that the database of known nuclei to the left of this cluster contains both important mass and shell correction information which were essential input for predictions in this mass region.

$Z=44$ to 48 , neutron rich; $A$ broad region from $Z=35$ to 48 on the neutron-rich side has mass model predictions with relatively small divergencies (frequently less than $1 \mathrm{MeV}$ ). Between the $\mathrm{Z}=$ 44 to 48 (squares in Figure 2) quite small divergencies occur. This seems to be associated with a grouping of nuclei that are thought to have small and slowing changing deformation. Adjacent known (fission product) nuclei have been well studied and many well determined masses have been made in this region. These features are presumed to aid accurate predictions for these nuclides.

$Z=37$ to 43 , along $N=Z_{i}$ There are several nuclides in this region, on or very close to the $N=Z$ line that have small spreads in their mass predictions. As one moves toward greater proton richness the divergencies grow rapidly (i.e. right pointing arrows, plus signs). Adjacent nuclei that do not appear in figure 2 have been studied via reactions like $40 \mathrm{Ca}+{ }^{40} \mathrm{Ca}$ and ${ }^{40} \mathrm{Ca}+58 \mathrm{Ni}$ and masses have been measured. Variants of these reactions with radioactive beams or targets should allow these presently unmeasured isotopes to be produced in useful yield for mass measurements (see below).

$Z=73$ to 76 , neutron rich: Within the $Z=62$ to 84 region shown in figure 4 there is a small cluster of neutron-rich nuclei with $Z=73$ to 76 that exhibit small divergencies in their predicted masses. It is interesting to note that this particular region is no longer accesible by fission and that just a few sparse new mass measurements in less neutron-rich isotopes have been made in the last decade or so. Thus any new measurements among these nuclei or adjacent ones would be quite useful here as tests of nuclear structure and mass models and to guide additional measurements in the future.

$Z=81$. startine near/bevond $N=126$; Figure 3 also shows an interesting feature for $Z=81$ (T1). The line of diamond symbols (rms deviations between 0.5 and $1 \dot{\mathrm{MeV}}$ ) that start just beyond $\mathrm{N}=126$ extends to the right much further than for adjacent elements. Eventually the trend breaks for $Z=81$ and divergencies in the mass predictions for very neutron-rich $\mathrm{Tl}$ isotopes mirror those of $\mathbf{H g}$ and $\mathrm{Pb}$. The "well behaved" nature of the double shell closure at ${ }^{208} \mathrm{~Pb}$ and a large well determined mass database for neutron-rich $\mathbf{H g}, \mathrm{Tl}$, and $\mathrm{Pb}$ isotopes (principally determined from the terminating members of the natural decay chains) are supporting aspects in the mass models that appear to yield consistent sets of predictions. What is unclear is why this is more prominent in the $\mathrm{Tl}$ isotopes.

$Z=86$. neutron rich and $Z=100$ to 104 , alons $N=156$ \& 157

As a general feature it should be noted that the mass prediction divergencies are rather gentle on both sides of stability in the heavy element region (Figure 5). Two areas within this region exhibit small divergencies in the mass model predictions. The first of these is in neutron rich radon $(Z=86)$ isotopes and it appears to be associated with the extensive nuclear structure and nuclidic mass databases of slightly less neutron-rich Rn isotopes that have been accumulated from studies of the natural radioactivity chains and studies of radon isotopes done with on-line separators. The second region occurs for $N=156$ and 157 in elements 100 to 104 . This feature appears to be connected to the fact that these nuclides represent the heaviest long-lived actinide isotopes and that extensive nuclear spectroscopy and mass measurements have been performed in this region that have fleshed out the mass surface and quantified the details of the microscopic nuclear structure. 
1.6.2 Regions where divergencies in mass prediction are large: mass regions to study with radioactive beams and/or targets

We now turn to those regions where divergencies in the mass predictions are large and/or grow rapidly on excursion from the stability line. In general each of these regions lie more remotely from stability than those discussed in the preceeding section. In almost all cases radioactive beams or targets will have to be used to reach these regions.

$Z=10$ to 20 . neutron rich: Inspection of figure 1 reveals that the predictions of the mass models are widely divergent in the light neutron-rich nuclei, ranging is some cases to rms deviations of more than $4 \mathrm{MeV}$. Any well measured masses of isotopes in this region will clearly by of great interest. The isotopes just beyond the known mass surface are already under study at the TOFI facility at Los Alamos 10 and the LISE spectrometer facility at GANIL 11. The advent of radioactive beam facilities that could capture, store, and cool light neutron-rich projectile fragments such as, for example, $40 \mathrm{~S}$ or $36 \mathrm{Si}$ from the projectile fragmentation of $48 \mathrm{Ca}$, will materially aid additional studies in this region. Inverse reactions of such beams on lighter targets are indicated.

$Z=28$ to 36 . neutron-rich, especially beyond $N=50$. For $N>50$ in the elements with $Z$ between about 28 and 34 one sees clusters of symbols (pluses, crosses, etc.) in figure 2 that indicate large divergencies in the mass model predictions. For $\mathbf{N}<50$ in the lighter elements of this region the divergencies are much smaller. This signals the influence of the $\mathbf{N}=50$ neutron shell closure. It also suggests that these nuclei, which are intimately involved in r-process nucleosynthesis beginning in lighter seed nuclei, are rather poor predicted. Careful mass measurements in this region could identify the model (or models) that best reproduce the mass surface. This could improve significantly the astrophysical aspects which are intimately connected to atomic masses. The best prospects for reaching these nuclei is by utilizing beams of projectile fragments from isotopes somewhat above this mass region, e.g. $96 \mathrm{Zr}$, $100 \mathrm{Mo}, 104 \mathrm{Ru}$; fission product 369 -day $106_{\mathrm{Ru}}$ also looks quite attractive.

$Z=44$ to 50 . along $N=Z ;$ Relatively few cases are represented in this region, shown in figure 2. However the few that are indicate that the small divergencies in mass model predictions that were characteristic of nuclei on or near the $N=Z$ line with $Z$ between 37 and 43 change to much larger ones for elements up to tin $(Z=50)$. The heaviest compound nucleus with $N=Z$ that can be made with stable beams and targets is $80_{\mathrm{Zr}}$ (via $40 \mathrm{Ca}+{ }^{40} \mathrm{Ca}$ ). Thus further studies of heavier $N=\mathrm{Z}$ nuclei via fusion-evaporation channels must employ radioactive beams and/or targets. Several candidate species that posses $N=Z$ and long lifetimes are ${ }^{44} \mathrm{Ti},{ }^{48} \mathrm{Cr}, 56 \mathrm{Ni}$. Each can be made in high yield by spallation of elements a few atomic numbers higher, conventional methods would be employed in either their acceleration or in the their fabrication as targets.

$Z=52$ to 95 , very neutron rich; Inspection of figures 3,4 and 5 for these nuclides shows a consistent pattern wherein the divergencies of the mass model predictions are small at the edge of the known surface but that they grow steadily. As the neutron drip-line is approached (right side cut off of plotted points at each $Z$ in figures 3 and 4 ) the divergencies have grown to more than $4 \mathrm{MeV}$. An equivalent statement is that the neutron drip line is poorly predicted throughout these elements. Most of the nuclei plotted in these figures will remain inaccessible even with the availability of radioactive beams or target. The ones that will be reachable however are well worth studying. If enough of them are characterized a pattern will develop that will identify the more successful mass models in this region. Some additional progress may be made using fast fission product separators or by extending fission product measurements into the low yield wings of the fission product distribution. Alternative methods to achieve adequate isotopic yields in these regions would be either 
projectile fragmentation or possibly the combination of very neutron-rich radioactive beams and very neutron-rich radioactive targets.

\subsection{Summary}

The analysis presented above has highlighted features of atomic mass predictions in regions beyond the experimentally known mass surface. Regions where predictions are generally similar have been noted. Other regions where atomic mass predictions diverge strongly from one another have been identified also. In the former cases modest extension of present day techniques commonly employed in studies of nuclear reactions and nuclear spectroscopy will permit the production and characterization of new isotopes. In the latter cases radioactive beams or radioactive targets (or both) will have to be employed to reach exotic new nuclides and study their properties. In both cases well determined nuclear properties such as masses will serve to test theory and to provide guidance for additional investigations.

\subsection{Acknowledgement}

This research was carried out at Brookhaven National Laboratory under Contract DE-AC02-76CH00016 with the U.S. Department of Energy and supported by its Division of High Energy and Nuclear Physics.

\subsection{References}

1. The 1986 - 1987 Atomic Mass Predictions, P. E. Haustein, Special Editor, At. Data Nucl. Data Tables 39, 185 (1988).

2. F. Tondeur, Self-consistent study of nuclei far from stability with the energy density method, in Proc. 4th Int. Conf. on Nuclei Far from Stability, P. G. Hansen and O. B. Nielsen, eds., CERN Report 81-09, Helsingor, Denmark, 1981, p81.

3. P. E. Haustein, A Comprehensive and Critical Review of the Predictive Properties of the Various Mass Models, in Proc. 7th Int. Conf. on Atomic Masses and Fundamental Constants (AMCO 7), 0. Klepper, ed., Technische Hochschule Darmstadt, Darmstadt-Seeheim, West Germany, p\$13, 1984.

4. P. E. Haustein, Am. Chem. Soc. Symp. Ser. 324, Nuclei Off the Line of Stability, R. A. Meyer and D. S. Brenner, eds. pp 126-131, 1986.

5. P. E. Haustein, Predictive Properties of Atomic Mass Models: Their Relationship to Nuclear Decay Modes by Spontaneous Charged-Particle Emission in Particle Emission from Nuckei, vol 1, D. N. Poenaru and M. S. Ivascu, eds., CRC Press, Boca Raton, FL, 1989.

6. 1975 Mass Predictions, S. Maripuu, Special Editor, At. Data Nucl. Data Tables, 17, 411 (1976).

7. P. Möller and J. R. Nix, At. Data Nucl. Data Tables 26, 165 (1981).

8. J. E. Monahan and F. J. D. Serduke, Phys. Rev. C17, 1196 (1978).

9. M. Uno and M. Yamada, Report INS-NUMA-40, Waseda University, Tokyo, Japan (1982).

10. J. M. Wouters et al. Los Alamos National Laboratory Report LA-UR-87-4293, 1987. 
11. A. C. Mueller et al. Z. Physik A330. 63 (1988).

Figure Captions

Figure 1. Root-mean-square (rms) divergencies of atomic mass predictions, plotted in the format of the Chart of the Nuclides for $Z=8$ to 30 . The rms deviations (in MeV) are binned by energy. Symbols that the bottom of the figure indicate the ranges of the energy bins.

Figure 2. Root-mean-square (rms) divergencies of atomic mass predictions, plotted in the format of the Chart of the Nuclides for $Z=26$ to 52 . See figure 1 for symbol definitions.

Figure 3. Root-mean-square (rms) divergencies of atomic mass predictions, plotted in the format of the Chart of the Nuclides for $Z=48$ to 66 . See figure 1 for symbol definitions.

Figure 4. Root-mean-square (rms) divergencies of atomic mass predictions, plotted in the format of the Chart of the Nuclides for $Z=62$ to 84 . See figure 1 for symbol definitions.

Figure 5. Root-mean-square (rms) divergencies of atomic mass predictions, plotted in the format of the Chart of the Nuclides for $Z=80$ to 108 . See figure 1 for symbol definitions. 


\section{MASS PREDICTIONS DIVERGENCIES}

$\mathrm{Z}=8 \mathrm{TO} 30$

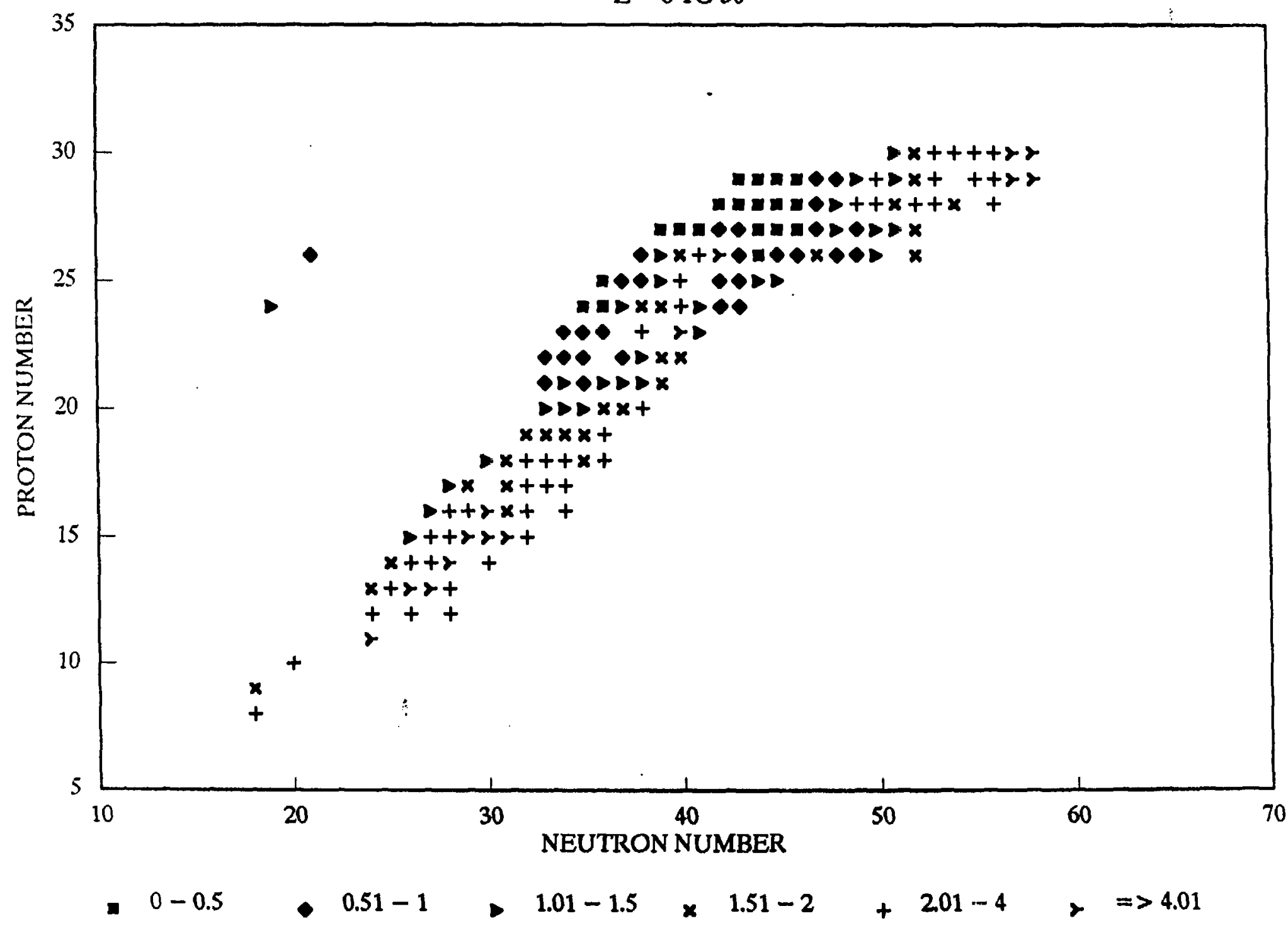

Haustem, figure 1 


\section{MASS PREDICTIONS DIVERGENCIES}

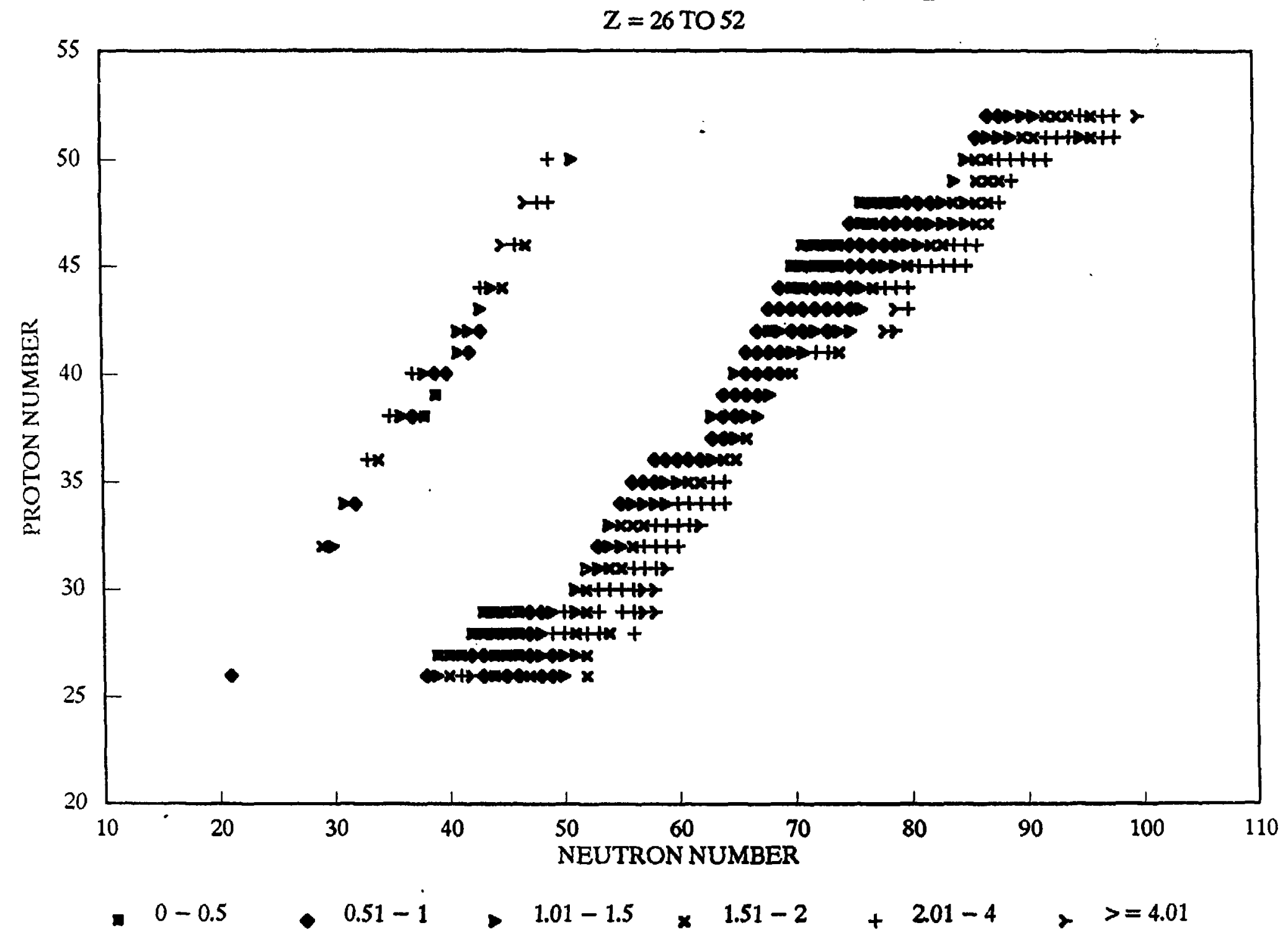

Hounstein, figure 2 


\section{MASS PREDICTIONS DIVERGENCIES}

$Z=48 \mathrm{TO} 66$

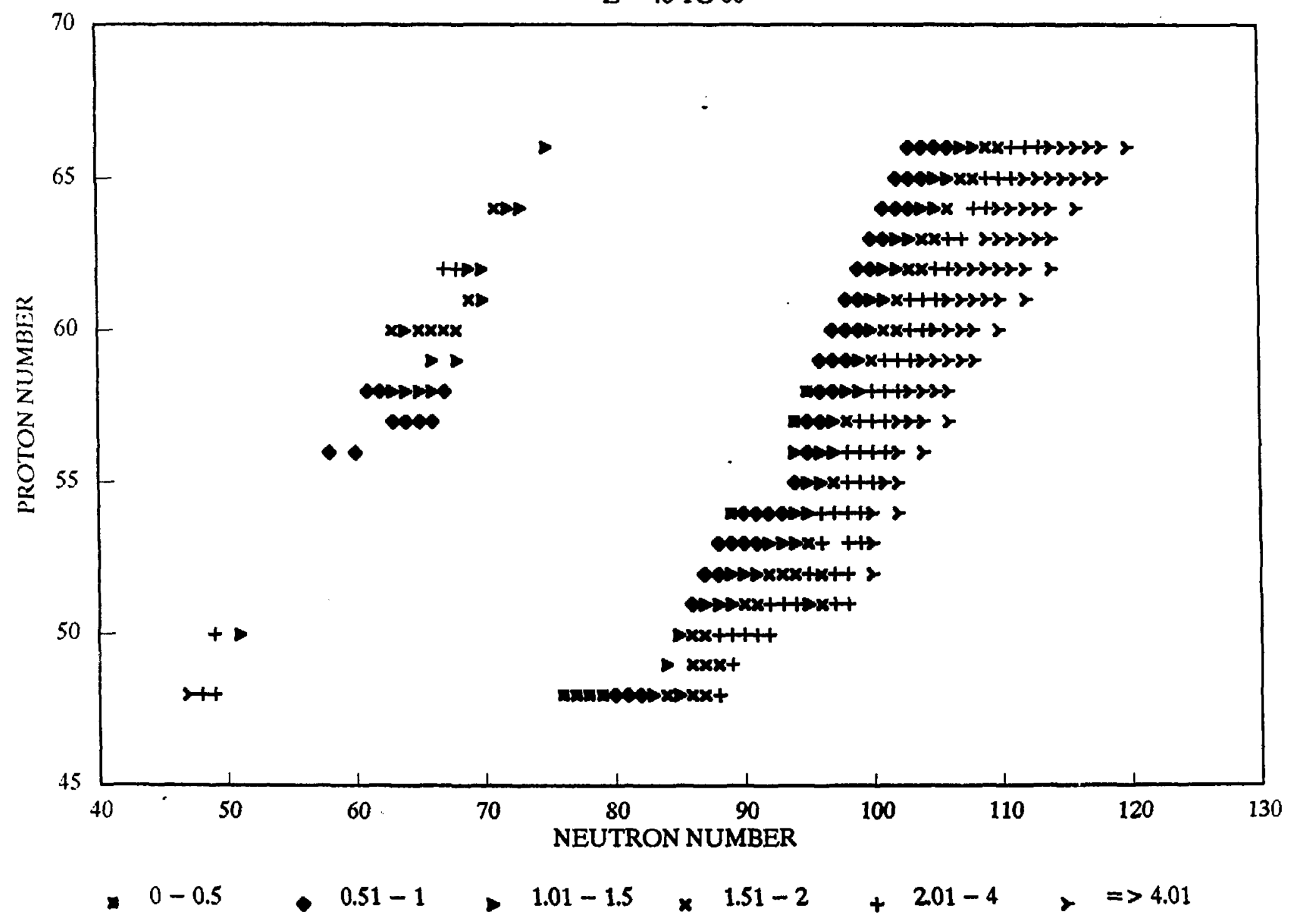

Hansterin. frecine 3 


\section{MASS PREDICTIONS DIVERGENCIES}

$$
\mathrm{Z}=62 \mathrm{TO} 84
$$

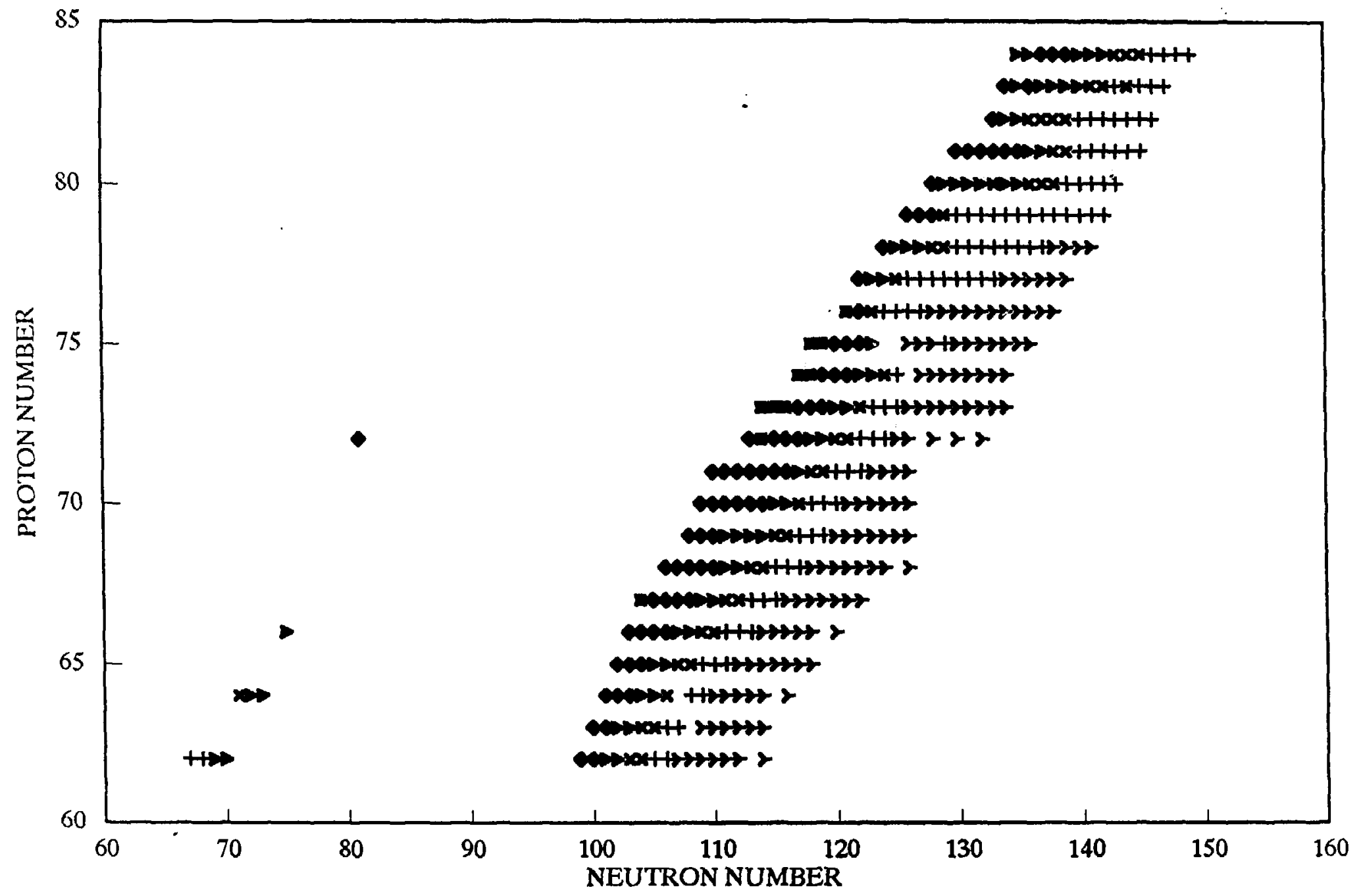

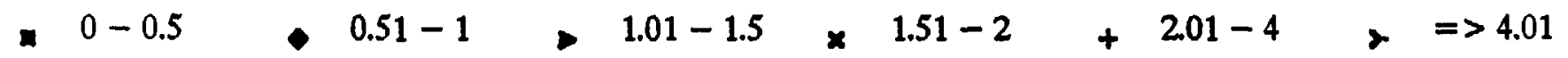




\section{MASS PREDICTIONS DIVERGENCIES}

$$
Z=80 \text { TO } 108
$$

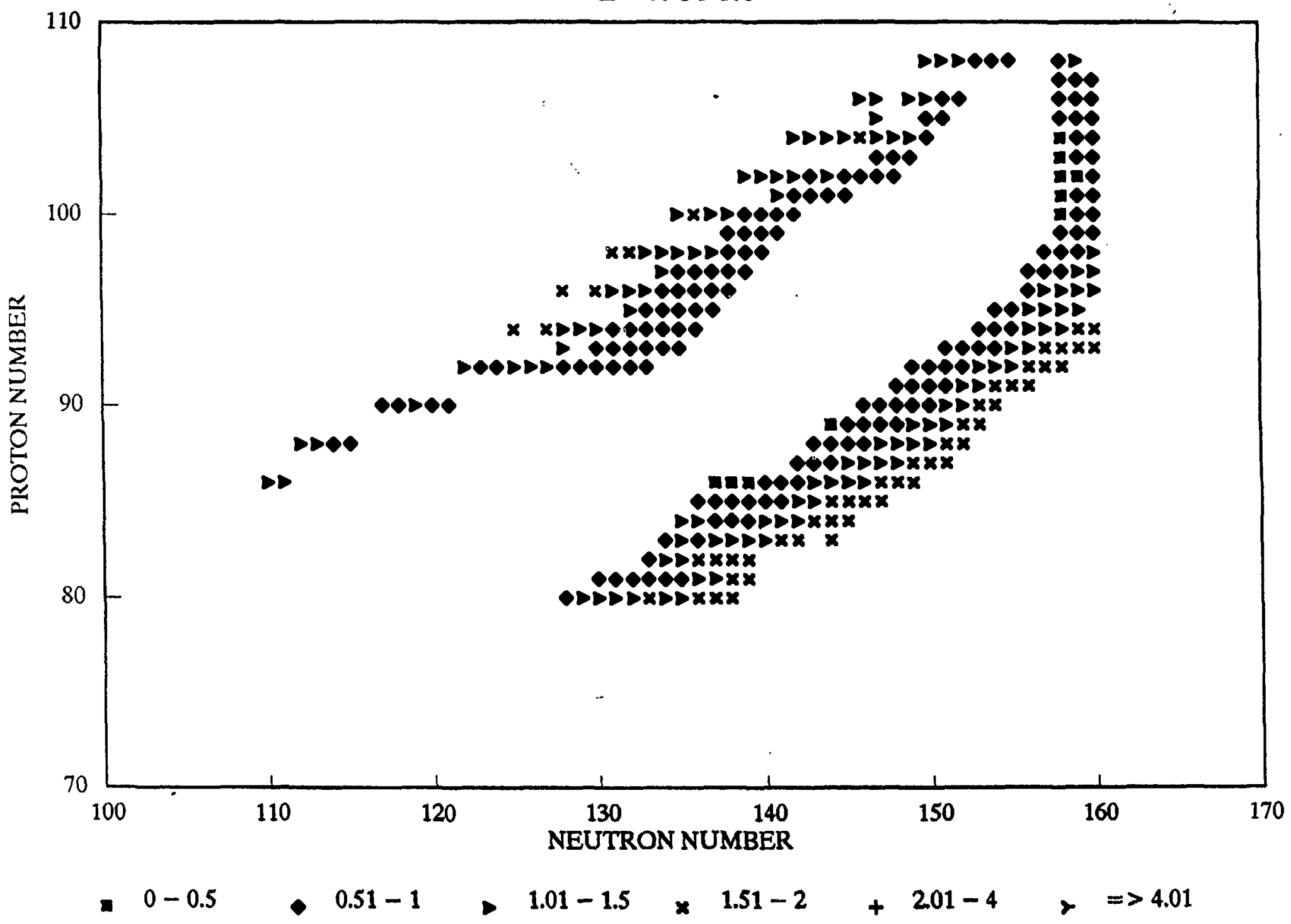

Hansteen, fyacire 5 